\title{
A multi-periodic oscillatory event in a solar flare
}

\author{
A. R. Inglis and V. M. Nakariakov
}

\author{
Centre for Fusion, Space and Astrophysics, Physics Department, University of Warwick, Coventry, CV4 7AL, UK \\ e-mail: a.inglis@warwick.ac.uk
}

Received 27 June 2008 / Accepted 14 November 2008

\section{ABSTRACT}

\begin{abstract}
Aims. Ratios of different significant periods found in the light curves of a solar flare exhibiting quasi-periodic pulsations (QPP) are used to distinguish between the possible physical mechanisms responsible for such periodic behaviour.

Methods. Time series data of the flaring event of 2002 July 3, observed via the Nobeyama Radioheliograph, Nobeyama Radiopolarimeters, and the RHESSI satellite, are investigated with the use of the Lomb-Scargle periodogram technique. Images of the event are also recovered via the RHESSI, SOHO, and Nobeyama Radioheliograph instruments.

Results. Statistical analysis of the Lomb-Scargle periodogram results indicates three distinct periods above the $99 \%$ confidence level in Nobeyama Radioheliograph and Radiopolarimeter data, at $28 \mathrm{~s}, 18 \mathrm{~s}$, and $12 \mathrm{~s}$, respectively. The two longest of these periods were also observed in the RHESSI data at the same confidence level. Wavelet analysis demonstrated that multiple periods occurred simultaneously without any significant frequency shift over time. Reconstructed images of the event reveal a very compact flare structure unsuitable for spatially resolved analysis. Consideration of the period ratios leads to the conclusion that the cause of this multi-periodic event is likely to be a kink mode periodically triggering magnetic reconnection.
\end{abstract}

Key words. Sun: flares - Sun: oscillations - waves - Sun: corona - magnetohydrodynamics (MHD)

\section{Introduction}

Quasi-periodic pulsations (QPP) are a regularly observed phenomenon in solar and stellar flare emission. In a typical event, the emission intensity from a flare is observed to oscillate with a characteristic period, which ranges from a fraction of a second up to several minutes ${ }^{1}$. QPP have been observed in the light curves of all EM bands associated with flare-generated emission, from radio to gamma rays - see Nakariakov (2007) for a recent review. Also, QPP are sometimes manifest not as intensity variations, but as periodic Doppler shifts in a flare emission spectrum (Mariska 2006). Our interest in flaring QPP is mainly connected with the additional constraints put on the physical mechanisms responsible for flaring energy releases by the oscillations, and also with the possibility of performing diagnostics of the physical parameters of plasmas in and near flare sites.

In general, an observed periodicity can be linked either with some oscillatory process connected with the elasticity or compressibility of the medium, with the release of energy by a periodic or aperiodic driver, or a combination thereof. In the first case, the observed period is determined by the geometrical scales of the oscillating structure, e.g. by the size of the resonator for a standing wave (Nakariakov \& Verwichte 2005) or by the waveguide width for a dispersive wave train (Nakariakov et al. 2004). In the second case, the period can be connected with the rate of energy supply to the system and by the threshold of onset of some dynamical process in the system, linked with the energy. Both options can be associated with QPP in flares. Indeed, flaring QPP can be linked either to MHD oscillations in the flaring region or in an external coronal plasma structure (Nakariakov 2007), or be connected with periodic regimes of magnetic

\footnotetext{
1 See the online catalogue

http://www2 . warwick.ac.uk/fac/sci/physics/research/ cfsa/people/inglisa/flarecatalogue
}

reconnection (Kliem et al. 2000; Ofman \& Sui 2006), or perhaps with some combination of these mechanisms. In both cases, the oscillations provide us with a powerful tool for plasma diagnostics, and for the determination of the physical processes operating in flares. QPP in thermal emission, e.g. in the soft X-ray band, can be directly caused by MHD oscillations of the emitting plasma, leading to periodic variations of the emission intensity and Doppler shifts or broadening of emission lines. The modulation of non-thermal emission (e.g., hard X-ray or microwave) can also be caused by MHD oscillations, either via modulation of the magnetic field in the emitting plasma, or by affecting the non-thermal electron dynamics. For example, this could mean the modulation of the electron trapping conditions, or the modulation of the non-thermal electron production rate, e.g. by periodic triggering of magnetic reconnection (Nakariakov et al. 2006). The latter can also be a self-induced periodic dynamical regime of magnetic reconnection, caused for example by the periodic creation of plasmoids.

Different mechanisms for the generation of QPP are differently manifested in observations. In particular, QPP which are observed to be simultaneous and in-phase in microwaves and hard X-rays are likely to be associated either with the sausage mode, because of the Zaitsev - Stepanov mechanism (Zaitsev \& Stepanov 1982) which produces periodic precipitation of nonthermal electrons, or with periodic reconnection which periodically accelerates the electrons. The variety of possibilities makes the identification of the specific mechanism responsible for an observed QPP event a non-trivial task.

The evolutionary nature of flares dictates that the total duration of QPP is usually short, rarely consisting of more than four or five complete cycles of the oscillation. As such, robust analysis of such events has proven difficult. In recent years advances in solar imaging and spectroscopy have made concrete analysis of solar oscillations a more realistic possibility. 
Melnikov et al. (2005) and Grechnev et al. (2003) have made first attempts at spatial resolution of solar flare QPP observed in microwaves. The spatial information allows one to distinguish between different mechanisms responsible for the observed QPP. However, even in the solar corona spatial information is not always available. In the stellar context this information is absent in all cases. Important information can also be provided by the multi-wavelength approach, when QPP is observed in different bands simultaneously (Melnikov et al. 2005; Inglis et al. 2008). Useful information can be provided by microwave or $\mathrm{X}$-ray spectral resolution (Fleishman et al. 2008), which is not always available.

In addition, the detection of several periodicities in the same signal can provide us with valuable knowledge. Different periodicities can either be associated with different MHD modes of the emitting plasma structure, or with different temporal harmonics of the same nonlinear oscillation. In both cases, the ratios of different periods can be used to reveal the physical mechanism responsible for the pulsations and hence become a tool for diagnostics (Andries et al. 2005). There have been several studies revealing the simultaneous presence of several periodicities in flaring light curves. For example, Stepanov et al. (2004) found $2.4 \mathrm{~s}$, $7 \mathrm{~s}$, and $14 \mathrm{~s}$ oscillations in the flare observed on 1999 August 28 using the Nobeyama Radioheliograph (NoRH); Melnikov et al. (2005) detected periodicities of 8-11 s and 14-17 s in the flare on 2000 January 12 observed with NoRH and Yohkoh/HXT; the study of Mészárosová et al. (2006) revealed the presence of multiple periodicities in the range from one to five minutes in the flares observed on 2000 July 14, 2001 April 12, and 2001 April 15 in radio $(0.8-4.5 \mathrm{GHz})$ and $\mathrm{X}$-ray bands using the Ondrejov radiospectrograph and Yohkoh/HXT. The traditional technique for the detection of multiple periodicities is the search for significant peaks in the Fourier time spectrum. However, it is non-trivial to estimate the confidence level of the detection when there are multiple peaks in the spectrum, as the techniques for the estimation of the confidence level give the result applicable to the highest spectral peak only (see the discussion in Horne \& Baliunas 1986). To the best of our knowledge, there have not been attempts to use multi-periodic information in the determination of the mechanism for QPP.

The aim of this paper is to analyse a flaring QPP event which contains several periodicities, to estimate significance of different peaks in time spectra, determine the period ratios, and also demonstrate that ratios of different periods can contribute to the determination of the physical mechanism responsible for the periodic behaviour. The paper is organised as follows: in Sect. 2 the lightcurves of the flaring event are presented at multiple wavelengths and the fundamental nature of the flare is described. Section 3 details the time series analysis illustrating the multiperiodic nature of the event. In Sect. 4 we concentrate on the available imaging information for this event, using data from RHESSI, SOHO and the Nobeyama Radioheliograph. Finally, in Sect. 5 we discuss our findings in terms of the underlying mechanisms that may be responsible for the observed QPP.

\section{Analysis of integrated light curves}

The flare analysed here was a GOES X1.5 class event. It occurred on 2002 July 3 at 02:10 UT and was observed by the Reuven Ramaty High Energy Solar Spectroscopic Imager (RHESSI) and the Solar and Heliospheric Observatory (SOHO) satellites. It was also observed by the ground-based Nobeyama Radioheliograph and Radiopolarimeter in Japan, but was missed by the TRACE satellite. According to the lightcurves in X-ray

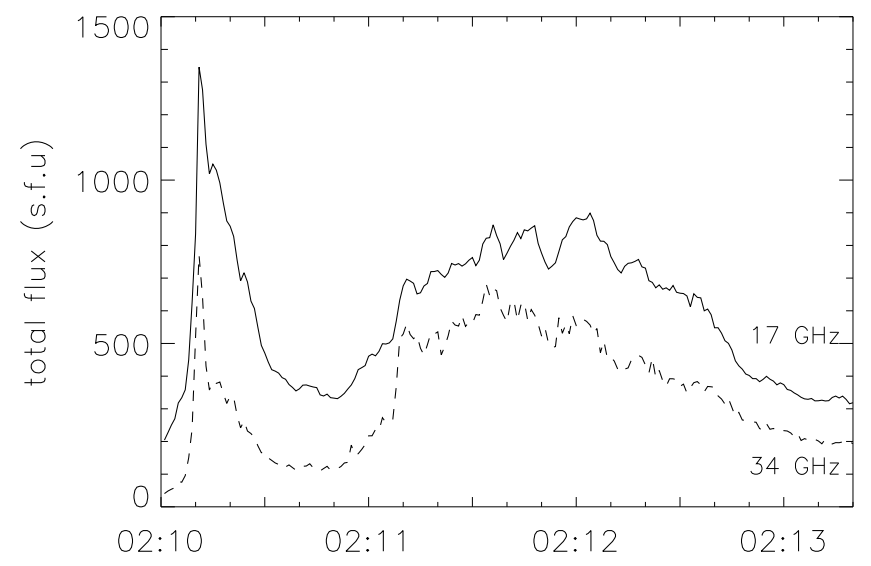

Fig. 1. Time profiles of the emission from the solar flare of 2002 July 3 , as observed by the Nobeyama Radioheliograph at $17 \mathrm{GHz}$ and $34 \mathrm{GHz}$. The first emission spike is seen just after 02:10, followed by an oscillatory phase between 02:11 and 02:13.

and radio wavelengths, the flare consisted of two distinct phases; a sharp initial energy release, followed shortly afterwards by a suspected oscillatory phase between $02: 11$ and $02: 13$. The period analysis presented here is concerned entirely with this second phase. This event is interesting in the context of this study as its time spectrum contains several pronounced peaks which are possibly connected with multi-periodic oscillations. Also, it was observed with good time resolution by three different instruments, which minimises possible instrumental artifacts.

The total flux time profiles of the radio emission from the entire event region, at the dual frequencies of $17 \mathrm{GHz}$ and $34 \mathrm{GHz}$ used by the Nobeyama Radioheliograph, are shown in Fig. 1. The time cadence of the signal is $1 \mathrm{~s}$.

The flux at $17 \mathrm{GHz}$ is stronger than that at $34 \mathrm{GHz}$, a typical observation for solar flares. The time profiles clearly illustrate a strong impulsive emission spike at approximately 02:10 UT. The emission drops off rapidly before rising again at 02:11 UT in what we will consider to be the second phase of the flaring event. The flux time profiles (light curves) during this second phase are suggestive of the presence of quasiperiodic pulsations. Similar quasi-periodic features are seen in the lightcurves obtained with the Nobeyama Radiopolarimeters at $9 \mathrm{GHz}, 17 \mathrm{GHz}$ and $35 \mathrm{GHz}$.

The emission time profiles for this event registered by RHESSI in different energy channels are shown in Fig. 2. Here the time resolution is $4.2 \mathrm{~s}$, coinciding with the spinning period of the RHESSI spacecraft. The flux levels shown in Fig. 2 have been arbitrarily scaled to avoid overlap and preserve the clarity of the temporal behaviour. The comparison of microwave and $\mathrm{X}$-ray fluxes shows that the same behavioural trend is exhibited by both the microwave and X-ray time profiles, i.e. an initial spike followed by a second event phase. This is significant as $\mathrm{X}$-rays and microwaves are generated by different mechanisms, and implies that some additional common physics is present. In the X-ray data, there is also an attenuator state change at the flare onset, visible as a jump in X-ray counts at approximately $02: 10: 10$. This is the result of an automated process whereby shutters are activated in front of the RHESSI detectors to reduce count rates. This avoids problems associated with high detector dead-time and limited spacecraft memory.

The phase relationship between oscillatory signals observed with different instruments is important for understanding the underlying physics behind a QPP event. We begin with 


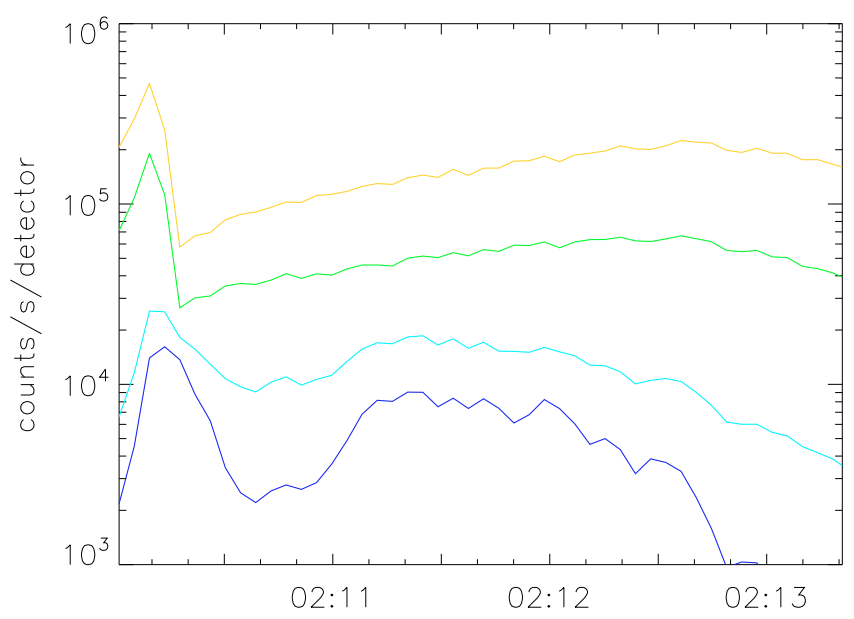

Fig. 2. Time profiles of X-ray emission from the solar flare of 2002 July 3, observed by the photometric detectors on board RHESSI. Yellow top: 6-12 keV emission, green: $12-20 \mathrm{keV}$ emission, light blue: 20-40 keV emission, dark blue bottom: $40-60 \mathrm{keV}$ emission. To avoid overlapping the light curves have been scaled by arbitrary factors.

consideration of the correlation between the light curves observed in microwave and X-ray bands, calculating the correlation coefficient. After removal of a background function, analysis of the time series showed a strongly in-phase relationship between $17 \mathrm{GHz}$ microwave data and the RHESSI counts at 20$40 \mathrm{keV}$ and $40-60 \mathrm{keV}$. The highest value of the correlation coefficient was 0.83 between the $17 \mathrm{GHz}$ signal and RHESSI counts at $40-60 \mathrm{keV}$, and corresponded to zero lag between the signals. Similarly, at $20-40 \mathrm{keV}$ the correlation value with the microwave signal was 0.67 , again corresponding to zero time lag. The same analysis showed that the RHESSI counts in the $12-20 \mathrm{keV}$ band and the $17 \mathrm{GHz}$ microwave data were largely uncorrelated. This is not surprising since the X-ray counts in this energy range are generally thermal in origin, while the signals in the other analysed bands are associated with nonthermal emission.

In order to investigate whether oscillations are occuring in this event, the time profiles must be carefully analysed. One of the most robust and commonly used techniques for this is the Lomb-Scargle periodogram method (Scargle 1982), which also provides estimates for the confidence level of spectral peaks as defined by Horne \& Baliunas (1986). In the context of solar flares, because of their short duration and evolutionary nature it is necessary to prepare the signal prior to utilising the periodogram. Spectral filtering is used to remove the lowest frequencies in the fourier spectrum, corresponding to the long term evolutionary trend of the flaring emission. The Lomb-Scargle periodogram is then performed on the remaining signal. It is important however that only the minimum necessary level of filtering is applied, and as much as possible of the original signal is preserved.

The Lomb-Scargle periodogram method was applied to the data obtained by the Nobeyama Radioheliograph during the second phase of the flare, between 02:11 and 02:13. The resulting periodogram is shown in Fig. 3.

A period of approximately $28 \mathrm{~s}$ is revealed with greater than $99 \%$ confidence in both the $17 \mathrm{GHz}$ and $34 \mathrm{GHz}$ channels. There are also other spectral peaks that may be significant, but require careful analysis. According to Horne \& Baliunas (1986) the significance levels of secondary peaks in a Lomb-Scargle periodogram are not accurately defined, since spectral leakage from the primary peak effects the remainder of the spectrum. In
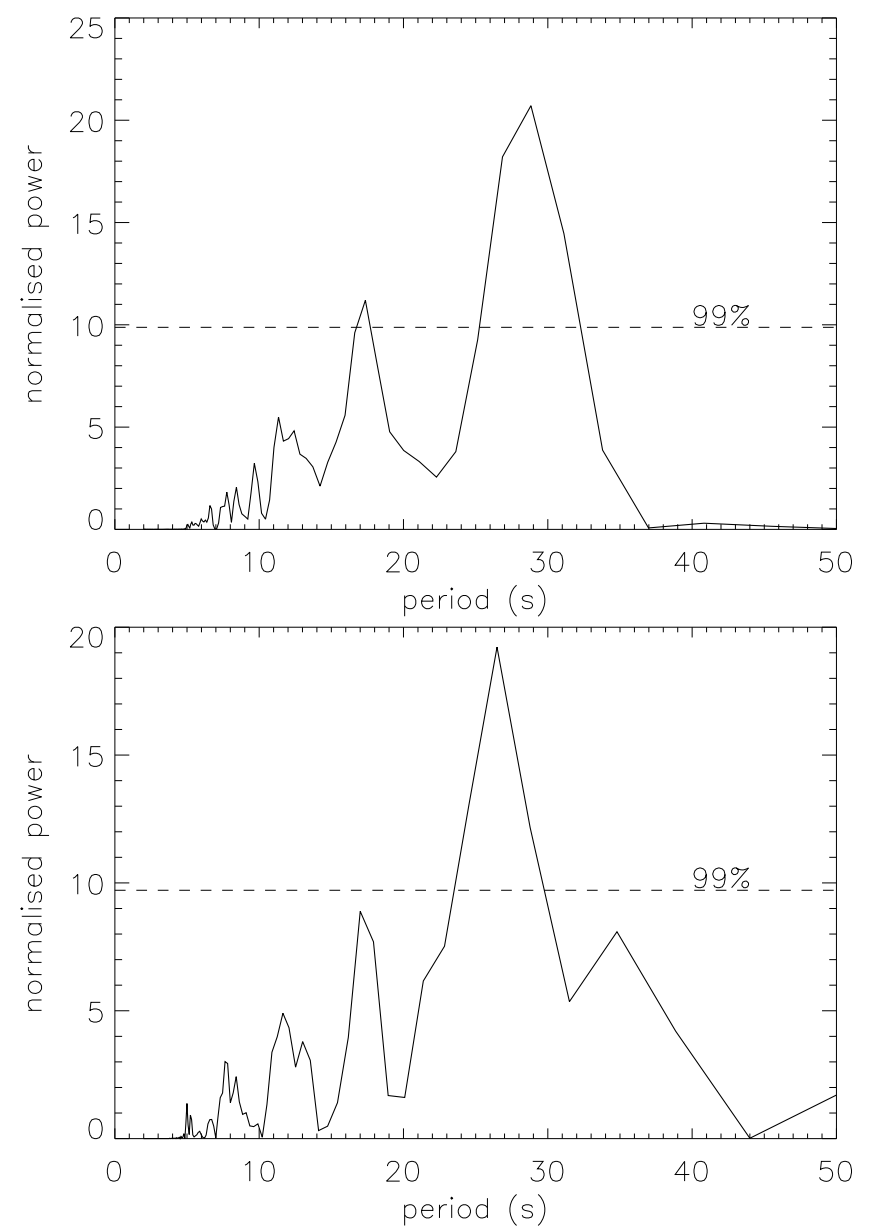

Fig. 3. Lomb-Scargle periodogram of the $17 \mathrm{GHz}$ (top) and $34 \mathrm{GHz}$ (bottom) signals from the Nobeyama Radioheliograph during the second flaring phase. The periodogram reveals a dominant period of $28 \mathrm{~s}$ at $17 \mathrm{GHz}$, well above the $99 \%$ confidence level defined by Horne \& Baliunas (1986). This period is also present in the $34 \mathrm{GHz}$ data above $99 \%$.

order to examine the significance of secondary peaks, this effect must be accounted for.

This technique can also be applied to RHESSI time series data. The RHESSI satellite rotates on its axis with a period of approximately $4 \mathrm{~s}$. This artificial period could have a significant impact on any frequency analysis technique. The simplest solution is to utilise the X-ray data with time bins of $4 \mathrm{~s}$, thus eliminating the rotation period from the data. If a greater number of datapoints are required it is also possible to employ shorter time bins. In this case the artificial period can be removed by spectrally filtering all periods of $4 \mathrm{~s}$ or less from the data.

The periodogram of RHESSI counts between 02:11 and $02: 13$ in the $6-12 \mathrm{keV}$ and $40-60 \mathrm{keV} \mathrm{X}$-ray ranges is illustrated in Fig. 4 for this event using time bins of $4.2 \mathrm{~s}$.

The Lomb-Scargle periodograms of the RHESSI data shows a peak near the $90 \%$ confidence level at roughly the same location as the primary Nobeyama Radioheliograph peak. In fact, the shape of the hard X-ray fourier spectrum is very similar to that obtained from the radio data. However, for the $17 \mathrm{GHz}$ and $34 \mathrm{GHz}$ spectra the peaks are substantially above the $99 \%$ confidence level. The lower amplitude RHESSI spectral peaks can be attributed to the reduced number of data points available for sampling. Nevertheless, this correlation between independent instruments at different energies is significant and should be explored. 


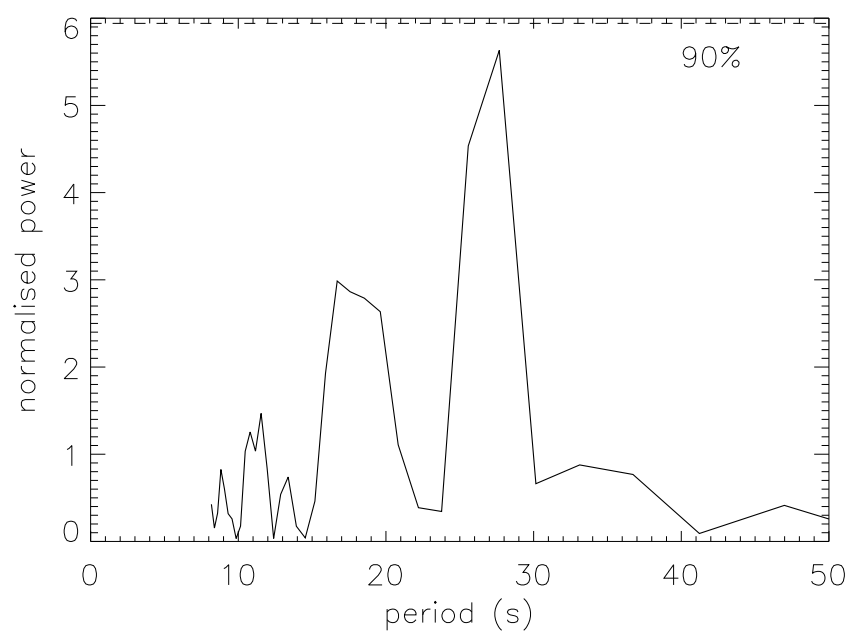

Fig. 4. Lomb-Scargle periodograms of RHESSI time series data in the 40-60 keV range using $4.2 \mathrm{~s}$ time bins. The dominant peaks are broadly consistent with those observed in Nobeyama Radioheliograph data.

\section{Analysis of multiple periods}

The presence of multiple significant peaks in a QPP event can shed light on the physical mechanism responsible for QPP. In both the Nobeyama and RHESSI periodograms shown in Figs. 3 and 4 there is a suggestion of more than one significant peak. Periodogram analysis is notoriously noisy (Scargle 1982), and the presence of multiple peaks must be analysed with care, as they may be artificial in origin. For example, spurious strong peaks may be generated due to sidelobes and spectral leakage from a genuine strong peak. Misapplication of spectral filtering may also cause peaks to be artificially enhanced.

The nature of secondary periodogram peaks may be tested. The technique is based on selective use of spectral filtering, whereby the principal peak is filtered out down to the background noise level. The periodogram is then recalculated on the remaining signal. Using this method one would expect genuine periods to increase or remain steady in spectral power, while artificial peaks would be reduced in power. This is because a strong primary peak can cause spectral leakage to other frequencies.

The Lomb-Scargle periodogram technique is a widespread and well-understood tool for time series analysis, in particular because there is a robust procedure for the estimation of the statistical significance of each spectral peak. As pointed out in Horne \& Baliunas (1986), the confidence levels in the Lomb-Scargle periodogram are only strictly valid for the most powerful peak in the spectrum. When this primary peak is carefully removed from the signal, the recalculated periodogram confidence levels are defined as the probability of a real signal being present at the secondary frequency, given that a signal already exists at the primary frequency.

This process can be iterated if there are multiple significant secondary peaks. The peaks are simply filtered in order of significance until no more peaks remain above the specified confidence level. The method is purely statistical however, and the onus is on the researcher to interpret the results. For example, in Muglach \& Balthasar (2005) it was noted that confident artificial oscillations in TRACE data were observed as a result of the satellite's image compression algorithms.

This analysis method was applied to $17 \mathrm{GHz}$ Nobeyama Radioheliograph data and Nobeyama Radiopolarimeter data at $9 \mathrm{GHz}, 17 \mathrm{GHz}$ and $35 \mathrm{GHz}$. The results of the iterative spectral filtering are shown in Fig. 5. The top panel of each column shows the original periodogram, while the subsequent panels show the effects of filtering the primary peak and recalculating the periodogram. The iteration process reveals the same three significant periods at multiple frequencies for two seperate instruments; $P_{1}=28 \pm 2 \mathrm{~s}, P_{2}=18 \pm 1 \mathrm{~s}$ and $P_{3}=12 \pm 1 \mathrm{~s}$. Here the error has been estimated using the peak half-widths shown in Fig. 5. The clear implication is that these peaks are real, rather than a result of spectral leakage.

To corroberate this result, the same technique was applied to the RHESSI hard X-ray light curves. The original periodograms of the RHESSI data did not reveal any periods significant to the 99\% confidence level. However, as a further test we re-bin the RHESSI time series into $2 \mathrm{~s}$ intervals to improve the resolution. To compensate for potential spacecraft rotation effects all periods of $5 \mathrm{~s}$ or less are then spectrally filtered from the data. Figure 6 shows the resulting iterations of the hard X-ray periodograms in the $40-60 \mathrm{keV}$ range. The dashed line in the top panel corresponds to the original periodogram obtained using $4.2 \mathrm{~s}$ time bins. From this the presence of both the $28 \mathrm{~s}$ and $18 \mathrm{~s}$ periodicities are revealed in hard X-rays. The $12 \mathrm{~s}$ period seen in the radio data is not detected at a statistically significant level in the RHESSI light curve.

The fact that the peaks illustrated here coincide in period with those detected using the original 4.2 s time bins brings confidence to the result. That they also coincide with the periods obtained via two other seperate instruments is a further indication that the RHESSI spectra are not compromised by instrumental artifacts.

Also of interest is whether the multiple harmonics co-exist in the same time range. The possibility that an oscillation period evolves over time - thus giving the impression of multiple harmonics - must be considered. To test this, Morlet wavelet analysis was employed (Torrence \& Compo 1998).

The top panel of Fig. 7 shows the Morlet wavelet of the $17 \mathrm{GHz}$ Nobeyama Radioheliograph signal after background subtraction. The short duration of this event dictates that the overall resolution of the wavelet in both period and time is poor. Although significant periods are present (in particular $28 \mathrm{~s}$ and $18 \mathrm{~s}$ ), it is also unclear from this plot whether the multiple harmonics are indeed co-temporal.

To study the time localisation of the three detected significant periodicities in more detail, we filtered out signals in the frequency bands determined by the periodogram analysis (namely $0.03-0,04 \mathrm{~Hz}, 0.045-0.065 \mathrm{~Hz}$ and $0.08-0.11 \mathrm{~Hz}$ ), synthesised separately the three narrowband signals, normalised each of them to its maximum amplitude, and then summed up the three narrowband normalised signals into a new signal. This approach allows us to amplify the signals of the second and third spectral peals, making them more pronounced in the wavelet spectrum. Clearly, this method does not spoil the spectral information only if there is no frequency shift across the boundaries of applied spectral filters, which is consistent with the wavelet spectrum shown in the top panel of Fig. 7. The wavelet spectrum of the resultant time signal is shown in the bottom panel of Fig. 7. The three significant periodicities detected in the periodogram are seen to occur simultaneously.

\section{Imaging}

Information useful for the interpretation of the observed QPP could be obtained from imaging observations. The Nobeyama Radioheliograph and the RHESSI satellite are both equipped with imaging capabilities that allow this solar event to be clearly 

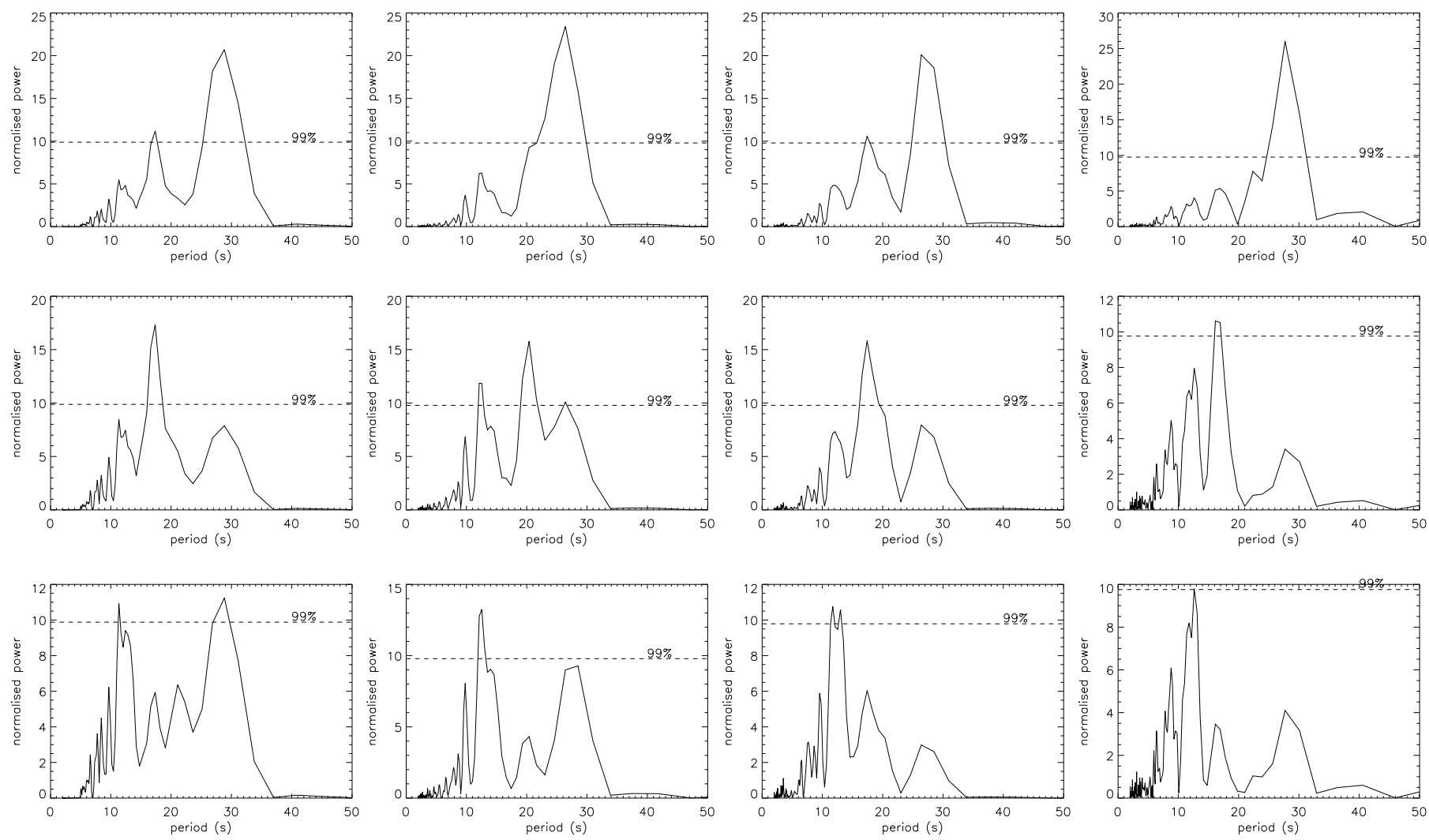

Fig. 5. Lomb-Scargle periodograms showing the effects of iterative peak suppression at different frequencies. Columns from left to right: Nobeyama Radioheliograph data at $17 \mathrm{GHz}$ and Nobeyama Radiopolarimeter data at $9 \mathrm{GHz}, 17 \mathrm{GHz}, 35 \mathrm{GHz}$. In all cases the same three peaks are found above the $99 \%$ confidence level.

resolved at various energies. In the case of the RHESSI satellite, collimating optics is used in order to reconstruct images of high energy emission, with a maximum resolution of approximately $2.5^{\prime \prime}$. The Nobeyama Radioheliograph is also capable of generating images, with a more modest maximum resolution of $5^{\prime \prime}$ at $34 \mathrm{GHz}\left(10^{\prime \prime}\right.$ at $\left.17 \mathrm{GHz}\right)$.

SOHO MDI images prior to the flaring event show the active region from which this flare originated. The image in Fig. 8 is taken around two hours before the onset of the flare. Based on this and Fig. 9, it appears that the primary emission source at both radio and X-ray wavelengths is centred between two sunspots.

Figure 9 contains a SOHO EIT image of this event with RHESSI overlays at thermal and non-thermal energies. The implication is that this was a consistently compact structure, with only a single clear emission source resolved. The 6-12 keV thermal emission is broadly consistent with the EIT $195 \AA$ structure, bearing in mind that different temperatures are observed and that the EIT image is necessarily taken before the onset of flaring activity because of image saturation during the flare itself. The 40-60 keV emission is non-thermal in origin, but appears to originate from the same source as the thermal component. In general, hard X-ray sources are expected at flaring loop footpoints and at the loop apex (Krucker \& Lin 2008), or occasionally above the loop apex (Masuda et al. 1994), while thermal $\mathrm{X}$-rays (such as the 6-12 keV range) are sourced from the body of the flaring loop. In this case it appears that any flare structure is too compact to be reliably resolved.
Microwave images from the Nobeyama Radioheliograph are also centred on this region. The main emission source at $34 \mathrm{GHz}$ is located at $(680,-360)$ arcsec, cospatial with the X-ray sources. Prior to the flare onset there is a second microwave source resolved at $(670,-330)$ arcseconds. This quiet region is dominated by the southern source during the flare itself. At $17 \mathrm{GHz}$, only a single source is visible, most likely due to limited spatial resolution.

Ideally any study of quasi-periodic pulsations in flares would include spatially resolved analysis. It has already been emphasised that knowledge of the spatial structure of an oscillation provides additional constraints on the physical mechanism responsible, and may also yield further information on various plasma parameters (Melnikov et al. 2005). Recently, Inglis et al. (2008) demonstrated the use of a cross-correlation mapping technique in an oscillatory solar flare from 8th May 1998. This method illustrates the cross-correlation values of the oscillatory signal component over different parts of the loop. It is also capable of revealing the phase relationship between different loop segments. Unfortunately, for the event of 2002 July 3 use of this method was compromised by instrumental effects, due to the strength of the signal. The Nobeyama Radioheliograph data is affected by jitter because of the high levels of flux from this flare. At peak flux levels the Radioheliograph is unable to properly resolve the solar disk, resulting in a loss of pointing accuracy. The spatial artifacts arising from this cannot be easily overcome. The pointing system of the RHESSI satellite proved more robust than that of the Nobeyama Radioheliograph in this case. However, 

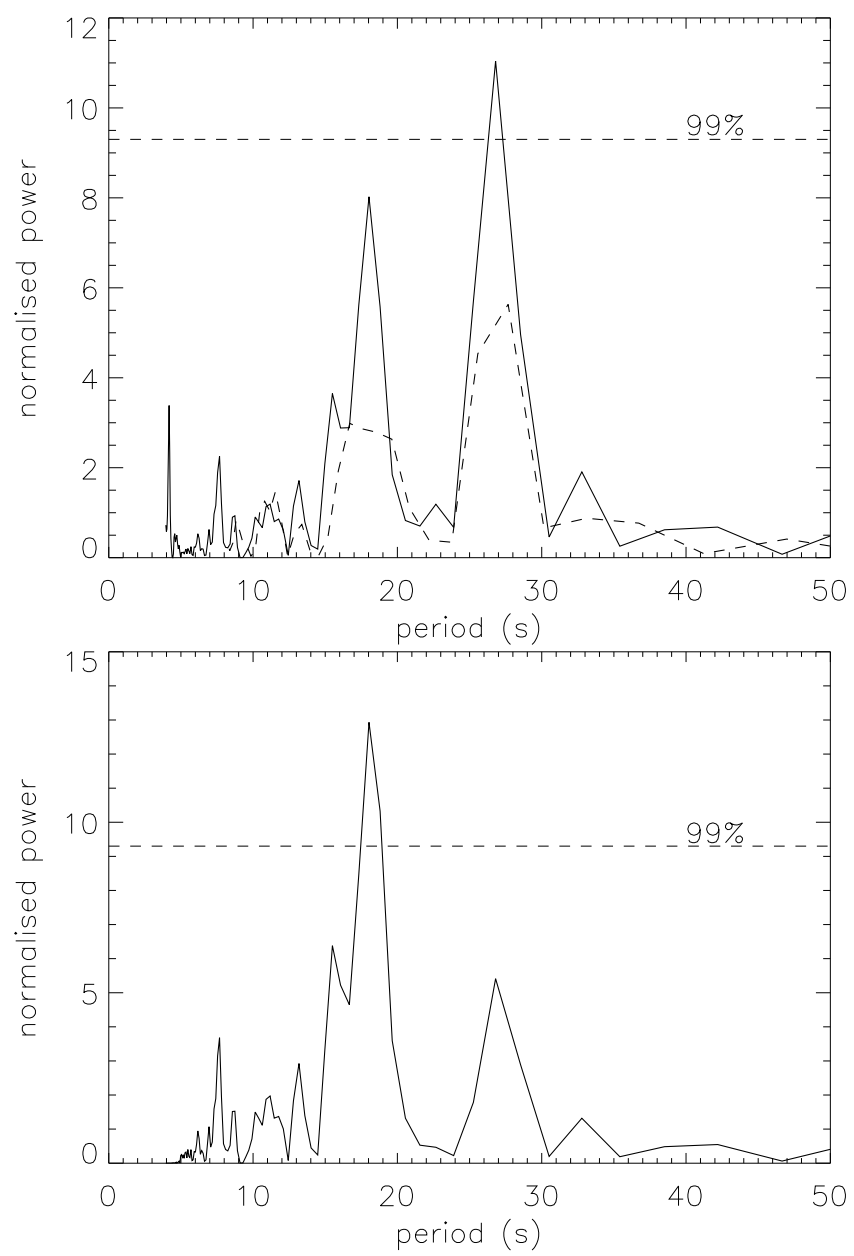

Fig. 6. Iterated Lomb-Scargle periodograms of RHESSI time series data between $40-60 \mathrm{keV}$ using $2 \mathrm{~s}$ bins. Top panel: original periodogram, showing the dominant $28 \mathrm{~s}$ period at $99 \%$ confidence. The original $4.2 \mathrm{~s}$ periodogram is shown as a dashed line for comparison. Bottom panel: periodogram after suppression of the $28 \mathrm{~s}$ peak. The $18 \mathrm{~s}$ period is now resolved above the $99 \%$ confidence level.

for the hard X-ray sources, no evidence of an extended spatial structure was found using cross-correlation analysis. RHESSI imaging relies on collimating optics and complex image reconstruction algorithms which may limit the applicability of the cross-correlation mapping technique. It is also possible that the emitting region is simply too small to be reliably mapped.

\section{Discussion}

In this study we analysed a multi-periodic flaring event and estimated the significance of different spectral peaks in the time spectra. Pulsations were observed during the second phase of the analysed flaring event in microwave and in a range of hard $\mathrm{X}$-ray bands. Although initial study indicated only a single period, careful analysis revealed that three distinct periods were significant above $99 \%$ confidence in the microwave data, obtained with NoRH and NoRP: $P_{1} \approx 28 \pm 2 \mathrm{~s}, P_{2} \approx 18 \pm 1 \mathrm{~s}$ and $P_{3} \approx 12 \pm 1 \mathrm{~s}$. The $28 \mathrm{~s}$ and $18 \mathrm{~s}$ oscillations were also detected in the RHESSI lightcurves in the 40-60 keV energy range. The $12 \mathrm{~s}$ period was not detected in the X-ray flux. We postulate that this is due primarily to the reduced temporal resolution of the X-ray data, a consequence of RHESSI spacecraft rotation.
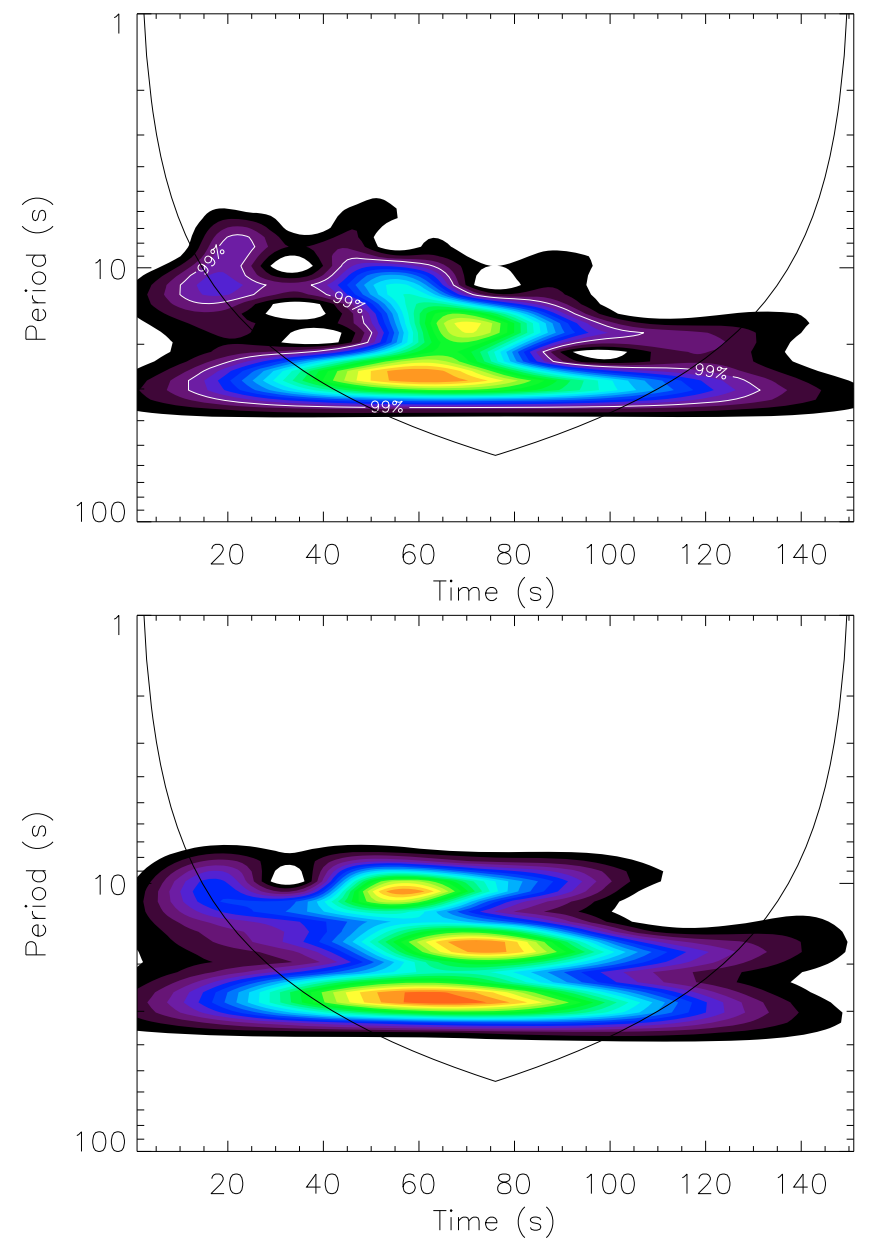

Fig. 7. Top: morlet wavelet of the $17 \mathrm{GHz}$ Nobeyama Radioheliograph signal after background subtraction. Bottom: a combined plot showing the result of taking wavelets in three frequency bands, corresponding to $P_{1}, P_{2}$ and $P_{3}$. The start time is $02: 10: 50$.

The presence of multiple significant periods in solar flare pulsations is a significant finding and poses interesting questions about the theoretical mechanisms that may be responsible. The fact that microwaves and hard X-rays share the same oscillatory behaviour is also important; microwave emission is generated mostly through the gyrosynchrotron mechanism, while hard $\mathrm{X}$-rays usually arise via non-thermal brehmsstrahlung and particle interactions at the photosphere. The presence of multiple periods in light curves obtained via different instruments also ensures that the periods are not instrumental artifacts.

Spatially resolved analysis of the flaring oscillation was not possible on this occasion, due to instrumental problems. However, event images show that the spatial extent of this flaring structure is very limited in any case.

Due to the lack of the information about the spatial structure of the oscillation, the interpretation of the observed oscillation should be based upon the analysis of the ratios between different periods, $P_{2} / P_{1} \approx 0.64 \pm 0.07$ and $P_{3} / P_{1} \approx 0.43 \pm 0.06$. If the multiple periodicity is caused by temporal harmonics of a nonlinear oscillations, because of the nonlinear doubling of frequency the resultant spectrum should be equidistant, i.e. the frequencies corresponding to different peaks are divisible by integer. This corresponds to the ratios of periods $P_{N} / P_{1}=1 / N$, where $N$ is an integer representing the harmonic number. The ratios of the lowest harmonics are $P_{2} / P_{1} \approx 0.5$ and $P_{3} / P_{1} \approx 0.33$. 


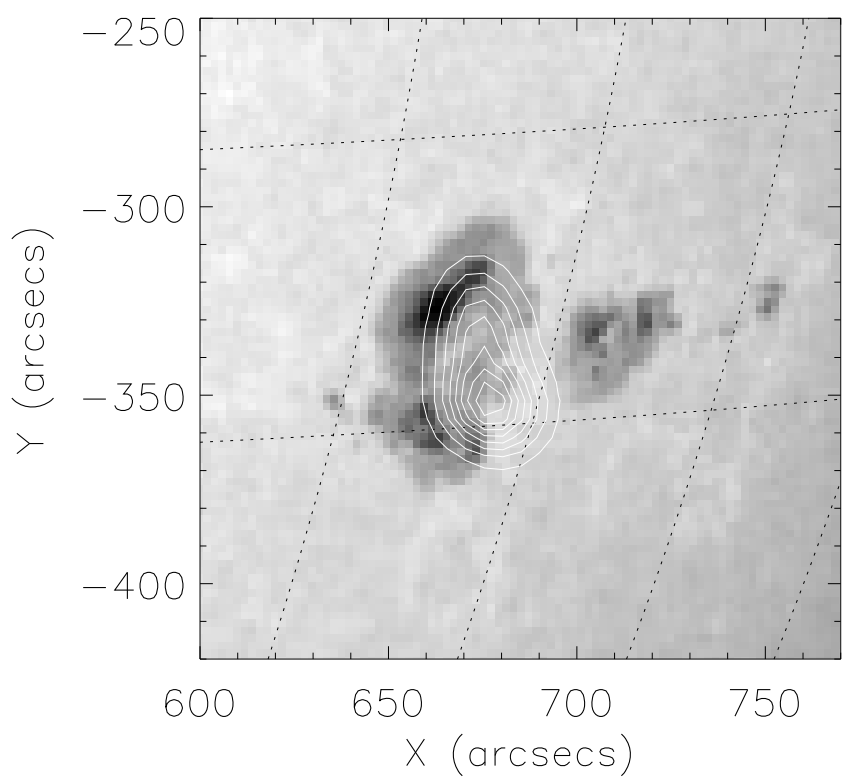

Fig. 8. SOHO MDI image of the active region from which the solar flare originated. This MDI image was taken at 23:59:00 on 2002 July 2. Overlay: Microwave emission at $17 \mathrm{GHz}$ during the flare, observed by the Nobeyama Radioheliograph.

Such a spectrum is a characteristic feature of an aharmonic oscillation, for example the periodic generation of plasmoids by magnetic reconnection (Kliem et al. 2000). Thus, we can see that the observed $P_{2} / P_{1}$ ratio is not exactly consistent with the $1 / N$ pattern. A certain discrepancy could be attributed to the error in the determination of the spectral peak position, however the error of about $30 \%$ is perhaps a bit too large. Thus, the interpretation of the observed periodicities in terms of a oscillatory regime of magnetic reconnection does not seem to be consistent with our findings. Another option could be the presence in magnetic reconnection of several simultaneous but independent oscillatory processes. However, we are not aware of any theoretical or numerical predictions of such an effect. Hence we exclude this interpretation of the observed event.

Similar $P_{N} / P_{1}$ ratios appear if the frequencies are connected with different spatial harmonics of a resonator, e.g. a coronal loop. However, in this case the ratios deviate from the $1 / N$ pattern because of dispersion, as waves with different wave numbers have different phase speeds, and the ratio becomes $P_{N} / P_{1}=$ $\left(V_{1} / V_{N}\right) / N$, where $V_{1}$ and $V_{N}$ are the phase speeds of the global mode and the $N$-th harmonics, respectively. We exclude the longitudinal and torsional modes of a magnetic flux tube from consideration as they are not able to produce the observed modulations of the microwave and hard X-ray emission simultaneously (Nakariakov 2007). Two other modes, sausage and kink, are able to produce the observed modulation: the sausage mode by the Zaitsev - Stepanov mechanism (Zaitsev \& Stepanov 1982), and the kink mode by the periodic triggering of reconnection (Nakariakov et al. 2006). Both sausage and kink modes of a plasma cylinder are known to be dispersive (Edwin \& Roberts 1983). For both sausage and kink modes, the phase speed monotonically decreases with the decrease in the wavelength, making the $P_{N} / P_{1}$ ratio greater than $1 / N$. Taking the values of the period ratios determined in this paper, we obtain that $2 P_{2} / P_{1} \approx 1.13-$ 1.46 and $3 P_{3} / P_{1} \approx 1.1-1.5$ which gives the monotonic decrease in the phase speed within the error bar, consistent with the theory.
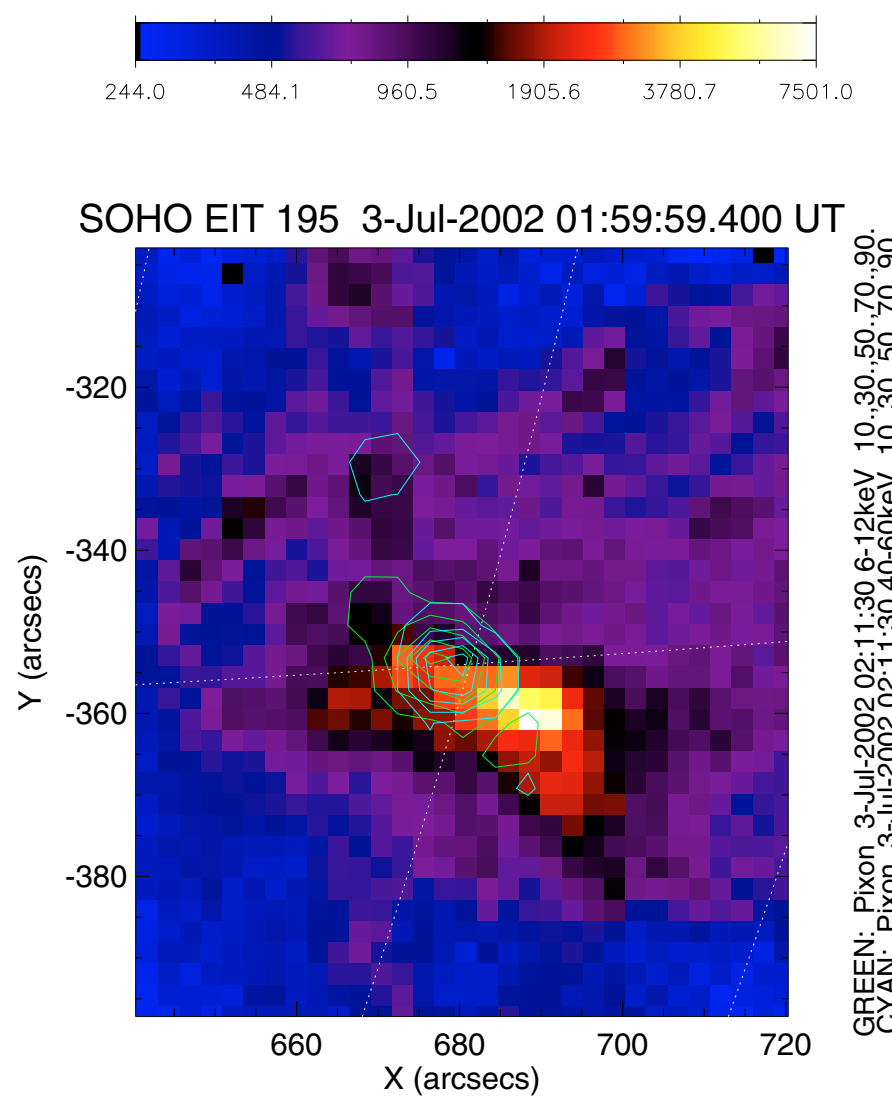

Fig. 9. SOHO EIT image of the flare region just prior to the onset of the flare. The overlays are RHESSI pixon reconstructions of X-ray emission during the oscillatory phase of the flare in the 6-12 kev (green contours) and 40-60 kev (cyan contours) bands.

Thus, both sausage and kink modes could produce the observed time spectrum. On the other hand, the observed lack of correlation between the light curves of thermal and nonthermal emission indicates that the observed periodic modulations are not likely to be produced by the sausage mode. Indeed, the sausage mode is an essentially compressible perturbation, and hence it should produce the modulation of thermal emission. The thermal emission produced by sausage waves is associated with the variation of plasma density caused by the oscillation of the magnetic field (through the frozen-in condition), and is then different from the change in the plasma density caused by field aligned flows of matter, e.g. chromospheric evaporation. Consequently, the variations of thermal emission induced by the sausage mode should be superimposed with the variations of the thermal emission, caused by evaporation. In our study this was not found. Thus, in our opinion the observed QPP should be interpreted as a multi-modal kink oscillation of an external coronal structure, which periodically triggers magnetic reconnection in the flare.

In this paper we made an attempt to identify the mechanism responsible for QPP generation based upon the assessment of the ratios of different periodicities found in the event light curves. This approach is necessary in cases where the spatial information is unavailable. As the same periodicities were detected in the data obtained with different instruments and in different bands, we claim confidently that these periodicities have natural origins in the solar corona. A similar approach can be applied to the interpretation of QPP in stellar flares (see for example Mathioudakis et al. (2003), Zaitsev et al. (2004) for 
recent findings), in which imaging information is intrinsically unavailable.

Acknowledgements. A.R.I. acknowledges the support of an STFC PhD studentship. The authors would like to thank Victor Melnikov (and acknowledge the Royal Society British-Russian Research Collaboration grant) for drawing our initial attention to this event. We would also like to thank Karin Muglach for helpful discussions. The authors are grateful to the Nobeyama and RHESSI teams and to Kiyoto Shibasaki and Brian Dennis personally.

\section{References}

Andries, J., Arregui, I., \& Goossens, M. 2005, ApJ, 624, L57

Edwin, P. M., \& Roberts, B. 1983, Sol. Phys., 88, 179

Fleishman, G. D., Bastian, T. S., \& Gary, D. E. 2008, ArXiv e-prints, 804

Grechnev, V. V., White, S. M., \& Kundu, M. R. 2003, ApJ, 588, 1163

Horne, J. H., \& Baliunas, S. L. 1986, ApJ, 302, 757

Inglis, A. R., Nakariakov, V. M., \& Melnikov, V. F. 2008, A\&A, 487, 1147

Kliem, B., Karlický, M., \& Benz, A. O. 2000, A\&A, 360, 715

Krucker, S., \& Lin, R. P. 2008, ApJ, 673, 1181

Mariska, J. T. 2006, ApJ, 639, 484
Masuda, S., Kosugi, T., Hara, H., Tsuneta, S., \& Ogawara, Y. 1994, Nature, 371, 495

Mathioudakis, M., Seiradakis, J. H., Williams, D. R., et al. 2003, A\&A, 403, 1101

Melnikov, V. F., Reznikova, V. E., Shibasaki, K., \& Nakariakov, V. M. 2005, A\&A, 439, 727

Mészárosová, H., Karlický, M., Rybák, J., Fárník, F., \& Jiřička, K. 2006, A\&A, 460,865

Muglach, K., \& Balthasar, H. 2005, in Chromospheric and Coronal Magnetic Fields, ed. D. E. Innes, A. Lagg, \& S. A. Solanki, ESA SP, 596

Nakariakov, V. M. 2007, Adv. Space Res., 39, 1804

Nakariakov, V. M., \& Verwichte, E. 2005, Living Rev. Sol. Phys., 2,3

Nakariakov, V. M., Arber, T. D., Ault, C. E., et al. 2004, MNRAS, 349, 705

Nakariakov, V. M., Foullon, C., Verwichte, E., \& Young, N. P. 2006, A\&A, 452, 343

Ofman, L., \& Sui, L. 2006, ApJ, 644, L149

Scargle, J. D. 1982, ApJ, 263, 835

Stepanov, A. V., Kopylova, Y. G., Tsap, Y. T., et al. 2004, Astro. Lett., 30, 480

Torrence, C., \& Compo, G. P. 1998, Bull. Amer. Meteorolog. Soc., 79, 61

Zaitsev, V. V., \& Stepanov, A. V. 1982, SvA Lett., 8, 132

Zaitsev, V. V., Kislyakov, A. G., Stepanov, A. V., Kliem, B., \& Furst, E. 2004, Astron. Lett., 30, 319 